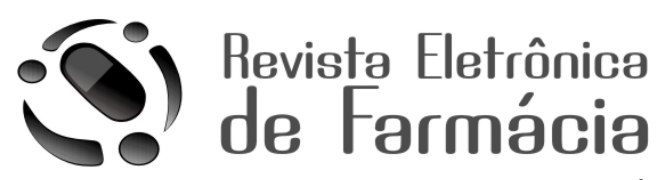

REF - ISSN 1808-0804 Vol. V (1), 8-23, 2008

\title{
ÁLCOOL E NEURODESENVOLVIMENTO: ASPECTOS GENÉTICOS E FARMACOLÓGICOS
}

\author{
Alcohol and Neurodevelopment: pharmacologics and genetics aspects
}

\author{
Deysi V. T. Wong ${ }^{1}$; Jose R. de O. Ferreira ${ }^{1}$; \\ Marta M. F. Fonteles ; Glauce S. B. Viana ${ }^{3}$; \\ Francisca C. F. de Souza ${ }^{3}$; Silvânia M. M. Vasconcelos ${ }^{3^{*}}$. \\ ${ }^{1}$ Mestrando. Pós-graduação em Farmacologia, Universidade Federal do Ceará, Brasil. \\ ${ }^{2}$ Docente. Faculdade de Farmácia, Odontologia e Enfermagem. Universidade Federal do Ceará. \\ ${ }^{3}$ Docente. Departamento de Fisiologia e Farmacologia. Universidade Federal do Ceará. \\ Rua Cel. Nunes de Melo, 1127. Fortaleza/Ceará - Brasil.
}

*Autor para correspondência e-mail: silvania_vasconcelos@yahoo.com.br

Recebido em 11/11/2007 - Aceito em 21/06/2008

RESUMO: O álcool, talvez, seja a droga mais antiga utilizada pela espécie humana, havendo relatos de seus usos em várias civilizações antigas como Grécia e Egito. O alcoolismo é uma síndrome complexa que envolve fatores ambientais, sociais, psicológicos e genéticos, sendo observado em $10 \%$ dos homens e cerca de $3-5 \%$ das mulheres, 10\% destas mulheres continuam a beber durante a gravidez. A Síndrome Alcoólica Fetal é uma condição que ocorre somente observada em crianças de mães que beberam durante a gravidez, acarretando aos recém nascidos malformação craniofacial, física e mental. O objetivo do trabalho foi realizar uma revisão de artigos envolvendo álcool e sistema nervoso central e suas implicações no neurodesenvolvimento, cognição e novos alvos terapêuticos, bem como modelos experimentais utilizados no estudo de drogas com ação no Sistema Nervoso Central. Diversos estudos, trabalhando com modelos animais de exposição pré-natal e pos-natal ao álcool, trazem resultados igualmente encontrados na Síndrome Alcoólica Fetal, como: déficits motores, aquisição de informação, formação de memória, dentre outros. Esses resultados confirmam os efeitos nocivos do álcool sobre o neurodesenvolvimento. Estudos com exposição pós-natal obtiveram os mesmos resultados quanto ao déficit motor e uma diminuição na neurogênese, bem como diminuição nos níveis de receptores D1 e D2, possivelmente causada pelo aumento nos níveis de dopamina. Estudos que tentam vasculhar o genoma de pessoas cuja família possui uma forte tendência ao alcoolismo procuram explicações moleculares para a dependência, trazendo consigo a descoberta de novos alvos farmacológicos que poderão agir de maneira sinérgica com as vias já descobertas (receptor opióide, aldeído desidrogenase, receptor GABA, Serotonina) ou serem mais eficazes e específicos.

PALAVRAS-CHAVE: alcoolismo, álcool, neurodesenvolvimento.

ABSTRACT: The alcohol is, perhaps, the oldest drug used by the human species, having stories of its uses in many ancient civilizations as Greece and Egypt. Alcoholism is a complex syndrome that involves environmental, social, psychological and genetic factors, being observed in $10 \%$ of all men and about $3-5 \%$ of all women, of these $10 \%$ continues to drink during pregnancy. The Fetal Alcohol Syndrome is a condition that occurs in children of mothers that had drunk during the pregnancy and it causes craniofacial, physical and mental malformation. The objective of this work was to carry out a review of articles involving alcohol and central nervous system and its implications in the neurodevelopment, cognition and new therapeutic targets, as well as of articles which have use experimental models 
to study the action of some drugs in the Central Nervous System. Very studies working with animal models of prenatal exposition to alcohol showed similar outcomes in relation to Fetal Alcohol Syndrome, as motor deficiency, decrease in acquisition of information and in memory formation, among other results, confirming the damage caused by alcohol in the neurodevelopment. Studies with postnatal exposition showed the same result related to motor deficiency and to a decrease in neurogenesis, as well as a decrease in D1 and D2 receptor levels, possibly caused by the increase of dopamine levels. Studies researching the genome of people whose family possess a strong trend for alcoholism try to find molecular explanations for the dependence, reaching the discovery of new pharmacological targets that will be able to act in synergic with the already discovered mechanism of action (opiate receptors, aldehyde dehydrogenase, GABA and Serotonin receptors) or to act more specific and efficiently.

KEYWORDS: Alcoholism, alcohol, neurodevelopmental.

\section{INTRODUÇÃO}

O álcool, talvez, seja a droga mais antiga utilizada pela espécie humana, havendo vestígios da sua existência desde períodos paleolíticos (WESTERMEYER, 1991). Na literatura médica, por volta do século 385 AC, Hipócrates descreveu o uso do álcool como um fator predisponente a várias doenças e relatou a respeito do delirium tremens em seu livro sobre as epidemias. No Egito e na Assíria, festas de veneração aos deuses da agricultura culminavam em bebedeiras coletivas que duravam dias seguidos. Conforme os Egípcios, o Deus Osíris teria ensinado os homens a cultivar a videira e a cevada para a fabricação de bebidas capazes de "inspirar a alma" (FERNADES \& FERNANDES, 2002).

Os dados epidemiológicos da Organização Mundial de Saúde (WHO) informam que o consumo de álcool é responsável por cerca de 1,8 milhões de mortes por ano (SEITZ \& STICKEL, 2007). Fora as pessoas que ficam incapacitadas quer seja por acidentes ou pelos efeitos lesivos do álcool sobre o organismo. O termo alcoolismo é usado para um transtorno marcado pelo uso crônico e excessivo do álcool, resultando em problemas psicológicos, interpessoais e médicos (FLEMING et al, 2006). Em 2000 os Estados Unidos tinha 20,687 mortes relacionados ao consumo de álcool, excluindo acidentes e homicídios, com um custo total estimado para a nação americana de cerca de US\$ 185 bilhões (NIAAA, 2000).

Já no Brasil, conforme o Centro de Informações sobre Drogas Psicotrópicas do Departamento de Psicobiologia da Universidade Federal do São Paulo - CEBRID, o uso de álcool em 108 maiores cidades do País é de 74,6\%, porcentagem inferior a de outros países como Chile (86,5\%) e EUA (82,4\%). O menor uso de álcool ocorre na Região Norte $(53,9 \%)$ e o maior na Região Sudeste $(80,4 \%)$. A estimativa de dependentes de álcool é de $12,3 \%$ (CEBRID, 2005).

Em vários países, o álcool é responsável por 30 a 50\% dos acidentes graves e fatais de trânsito (MARIN \& QUEIROZ, 2000; GAZAL-CARVALHO et al, 2002; HINGSON \& WINTER, 2003). O consumo desta substância tem sido associado á perpetração de $50 \%$ de todos os homicídios, mais de $30 \%$ dos suicídios e tentativas de suicídios, e uma ampla gama de comportamentos violentos (MINAYO \& DESLANDES, 1998; SPEAR et al., 2006). De acordo com MELCHIOR et al. (2008) entre 22 e 26 anos de idade 29,8\% dos homens e 15,6\% das mulheres fazem uso regular de álcool na França.

O consumo do álcool tem impacto nos diferentes sistemas orgânicos, por exemplo, no sistema nervoso central, no trato gastrintestinal, nos órgãos hematopoiéticos e no sistema imune (ZHOU et al., 2003; SCHUCKIT, 2005). O alcoolismo maternal durante os períodos pré e pós-natal contribui com alterações histológicas importantes nos órgãos linfóides dos animais descendentes (ARAUJO et al., 2007).

O objetivo deste trabalho foi realizar uma revisão bibliográfica sobre os efeitos do consumo de álcool no Sistema Nervoso Central e suas implicações no neurodesenvolvimento, cognição, aspectos genéticos e novos alvos terapêuticos.

\section{METODOLOGIA}

A fonte de pesquisa foi a través de livros, teses de doutorado e artigos científicos publicados na base de dados da PUBMED, SCIENCEDIRECT, projeto NIAAA (National Institute on Alcohol Abuse and Alcoholism) e REVISTA 
ELETRÔNICA DE FARMÁCIA. Utilizaram-se as palavras-chave Álcool "Alcohol", Alcoolismo "Alcoholism", Neurodesenvolvimento "neurodevelopmental"

\section{PROCEDIMENTOS ADOTADOS}

\section{Neurofarmacologia do Álcool.}

Um corpo extensivo de pesquisas indicou que o consumo crônico do álcool (isto é abuso e dependência) são associados com anormalidades na morfologia do cérebro, metabolismo cerebral de glicose e aminoácidos, sistema transmissor colinérgico e monoaminérgico, função e estrutura microcelular, fluxo sanguíneo cerebral regional e neurocognição (OSCAR-BERMAN, 2000; SULLIVAN, 2000).

O álcool é um depressor do sistema nervoso central, provocando uma desorganização geral da transmissão dos impulsos nervosos nas membranas excitáveis. Possivelmente, alguns dos seus efeitos são mediados por um mecanismo mais especifico envolvendo receptores glutamatérgicos - NMDA (N-metil-D-Aspartato) e gabaérgicos (GABA) (SCHUCKIT, 2005), sistemas serotoninérgicos (5-HT) (BOYCE-RUSTAY et al., 2006), opióides e dopaminérgicos (VASCONCELOS et al., 2004; VASCONCELOS S.M., 2001). Os efeitos pertubadores do álcool sobre o SNC são bem visíveis com a popular ressaca, que ocorre após a ingestão de doses tóxicas de álcool, caracterizada por efeitos adversos físicos e mentais (PRANT et al., 2008).

A exposição temprana ao etanol diminui o numero de neurônios em varias estruturas do Sistema nervoso central de ratos (MILLER, 1995; MOONEY, 1997). Em relação às propriedades físicas das membranas neuronais, vários estudos apontam para a capacidade do etanol alterar a estrutura das membranas lipídicas, aumentando a sua fluidez, dada sua propriedade lipofílica (RANG et al. 2007; KATZUNG, 2006). Entretanto, as conseqüências neurofisiológicas desta ação ainda não estão bem definidas (RANG et al. 2007). O consumo agudo e crônico do álcool e o catabolismo do etanol está associado com a geração de espécies reativas de oxigênio e outros produtos metabólicos, isso pode conduzir a dano oxidativo de estrutura e moléculas celulares, incluindo fosfolipídios e DNA (BROOKS, 2000).

Em relação à perturbação na atividade elétrica neuronal, sabe-se que altas doses de etanol inibem a atividade elétrica das células de Purkinge. O álcool etílico inibe a atividade de varias regiões cerebrais, como núcleo da rafe, oliva inferior, lócus cerúleos, hipocampo (MILLER, 1995; WEST et al., 1986; IERACl \& HERRERA, 2007), neurônios da região septal medial (SCHAMBRA et al., 1990). Entretanto, em determinadas áreas, como área tegmental ventral, substancia nigra (SHETTY et al., 1993) e núcleo accumbens, ricas em dopamina, o etanol aumenta a atividade espontânea, talvez por pura desinibição, já que o etanol é estritamente depressor. Provavelmente, esta ativação nestas regiões medeia às propriedades de reforço relacionadas à dopamina (KOOB, 1992). De uma forma geral, o etanol deprime a transmissão sináptica, interferindo, inclusive, com a plasticidade sináptica (DILDY-MAYFIELD \& HARRIS, 1995).

A atividade dos neurotransmissores, o álcool seletivamente altera a ação sináptica do glutamato no cérebro. O sistema glutamatérgico, que utiliza glutamato como neurotransmissor, é uma das principais vias excitatórias do sistema nervoso central, também parece desempenhar papel relevante nas alterações nervosas promovidas pelo etanol. O glutamato é o maior neurotransmissor excitatório no cérebro, com cerca de $40 \%$ de todas as sinapses glutaminérgicas (DIDLY-MAYFIELD \& HARRIS, 1995; FLEMING et al., 2006; SCHUCKIT, 2005).

O etanol reduz a atividade do receptor de glutamato NMDA (N-metil-D-aspartato) (SCHUCKIT, 2005; ROSSETI \& CARBONI, 1995), e inibe a produção do segundo mensageiro, GMPc, mediado pelo receptor. Com o uso crônico de etanol ocorre um aumento dos sítios de ligação do glutamato nos receptores NMDA, um "up-regulation" destes receptores, explicando a hiperatividade glutamatérgica que ocorre na síndrome de abstinência alcoólica (VASCONCELOS S.M, 2001). Estes resultados poderiam também participar da gênese de dependência física ao álcool, ou seja, uma vez retirado o etanol, as vias glutaminérgicas produzem superexcitação do SNC, gerando convulsões, ansiedade e delirium. Também foi postulado que esse receptor está envolvido em processos de aprendizagem e memória e no fenômeno da tolerância ao álcool (FERREIRA \& MORATO, 1997). Outros receptores de glutamato, o Kainato (GIBBS et al., 2000) e o AMPA, também sofrem a inibição induzida pelo consumo crônico de etanol, embora em menor escala do que o NMDA (DIDLY-MAYFIELD \& HARRIS, 1995). 
O etanol potencializa a ação do GABA, que é o principal neurotransmissor inibitório do Sistema Nervoso Central, atuando sobre os receptores $\mathrm{GABA}_{\mathrm{A}}$ de maneira semelhante aos benzodiazepínicos. Entretanto, seu efeito sobre os receptores é menor e menos consistente do que os benzodiazepínicos (RANG et al., 2007; GROBIN et al., 1998). As vias neuronais que utilizam GABA desempenham importante ação inibitória sobre as demais vias nervosas. O receptor para o GABA encontra-se associado ao canal de cloro e ao receptor de benzodiazepínicos, formando um complexo funcional (MIHIC \& HARRIS, 1997). Quando o GABA se acopla ao seu receptor, promove o aumento na freqüência de abertura dos canais de cloro, permitindo assim a passagem de maior quantidade do íon para o meio intracelular, tornando-se ainda mais negativo e promovendo, assim, hiperpolarização neuronal (GROBIN et al., 1998; AGUAYO et al., 2002; WHITTE et al., 2000). Com o uso crônico de etanol, ocorrem algumas alterações no sistema gabaérgico, a saber: (a)Redução da capacidade do etanol em estimular a ação do GABA, (b) Diminuição da capacidade de um agonista benzodiazepínico em potencializar a atividade gabaérgica, (c) Redução da densidade de mRNA relacionado ao local de ação dos benzodiazepínicos e etanol (AGUAYO et al., 2002).

As ações do etanol sobre o sistema dopaminérgico parecem ativar indiretamente vias serotoninérgicas, uma vez que podem ser atenuadas por antagonistas do receptor 5-HT3 (CARBONI et al., 1989; ALDERAZI \& BRETT, 2007). A relação entre etanol e receptores 5-HT3 também tem sido demonstrada em trabalhos centrados na teoria de que baixos níveis de 5-HT no cérebro podem ser um fator de risco para o alcoolismo (MATSUZAWA et al., 1999; MYERS \& VEALE, 1968).Estudos fármaco-comportamental mostram que muitos efeitos do álcool são mediados pela interação entre 5-HT e dopamina no sistema cortico-mesolimbico (BARNES \& SHARP, 1999).

O álcool etílico também afeta os canais de cálcio em concentrações fisiologicamente relevantes. O influxo de íons cálcio para a célula desempenha importante função na liberação dos neurotransmissores na fenda sináptica como também, na atividade de segundo mensageiro celular. O etanol, em concentrações de $25 \mathrm{mM}$ parece inibir a passagem de cálcio através dos canais iônicos, diminuindo a liberação de neurotransmissores. O etanol influencia o fluxo de cálcio ( $\mathrm{Ca}++)$ através da membrana celular, reduzindo-o, no período de intoxicação, por uma ação nos canais de cálcio do tipo-L (ZHANG et al., 2000). No período de abstinência alcoólica, há um aumento do influxo de Ca++ através desses canais, contribuindo para seus sintomas. Esse efeito compensatório pode ser reduzido, em animais de laboratório, pela administração de antagonistas de canal de Ca++, como a nifedipina (KENNEDY \& LIU, 2003).

A administração crônica de etanol leva a um aumento compensatório da densidade desses canais, de forma similar ao que ocorre com os receptores NMDA. Uma vez que tais mudanças persistem no período de abstinência, com um aumento generalizado da atividade elétrica neste período, os canais de cálcio dependentes de voltagem também parecem ter uma importante contribuição para os sintomas da Síndrome de Abstinência do Álcool (LONGO et al, 2002).

O sistema opióide endógeno, especialmente através de sua interação com o sistema da via dopamina cortiçomesolimbica (CDMA), está envolvido na expressão dos efeitos de reforço do álcool (LEE et al., 2005; GIANOULAKIS, 1998; JOHNSON \& AIT-DAOUD, 2000). Há evidencias do aumento da atividade opióide após o uso do álcool etílico (MILLER, 1992; GIANOULAKIS, 1993). Em pacientes com uma historia familiar de alcoolismo, a ingestão de etanol esta associada a um concomitante aumento dose-dependente dos níveis de $\beta$ - endorfinas (GIANOULAKIS, 1996).

Estudos que avaliam funções cognitivas associam a ingestão crônica de etanol com a redução na concentração cerebral de acetilcolina, tanto em humanos quanto em ratos, causada por degeneração do tecido cerebral (SASAKI et al, 1995). Antagonistas de colecistocininas reduzem os efeitos convulsivantes da abstinência alcoólica em camundongos (SINGH \& WOODRUFF, 1992).

A exposição crônica ao etanol pode resultar em uma modificação na estrutura da proteína G estimulatória (Gs) ou alterar as interações entre as subunidades da proteína G. Essas alterações interferem na estimulação da adenilato ciclase e na produção de AMPc, e parecem estar relacionadas ao desenvolvimento da tolerância ao álcool (RABBANI \& TABAKOFF, 2001). No entanto, outros estudos sugerem que, não apenas um, mas múltiplos processos podem estar envolvidos na regulação da atividade de segundos mensageiros pelo álcool (GORDON \& DIAMOND, 1993).

E importante ressaltar que o próprio etanol é neurotóxico e, embora a desnutrição ou a deficiência de tiamina provavelmente desempenha um papel nas complicações do alcoolismo (p.ex. encefalopatia de Wernicke e psicose de Korsakoff), a maior parte de lesões cerebrais induzidas pelo álcool nos países ocidentais é atribuída ao próprio etanol (FLEMING et al., 2006; SCHUCKIT, 2005). 
Segundo pesquisas realizadas por DURAZZO et al., (2007) a combinação do consumo crônico de álcool e fumo de cigarro, foi associada a uma maior anormalidade de marcadores na síntese/recaptação da membrana celular e viabilidade neuronal. As anormalidades da morfologia do cérebro, metabólitos, fluxo de sangue e neurocognição observados em alcoólicos fumantes podem ser relacionados à exposição crônica de numerosas fontes de estresse oxidativo e de outros compostos nocivos encontrados no fumo do cigarro (DURAZZO et al., 2007; FRIEND et al., 2005).

\section{Álcool, desenvolvimento e cognição:}

O alcoolismo é uma síndrome complexa que envolve fatores ambientais, sociais, psicológicos e genéticos (pesquisado no site: www.niaaa.nih.gov), sendo caracterizado por um conjunto de problemas relacionados ao consumo excessivo e prolongada do álcool, interferindo com a vida pessoal, profissional, familiar, social e a saúde do indivíduo. O alcoolismo é observado em 10\% dos homens e 3-5\% das mulheres, contudo, mais mulheres e jovens tem bebido excessivamente, cerca de $10 \%$ das mulheres continuam a consumir bebidas alcoólicas durante a gravidez (ALDERAZI \& BRETT, 2007).

Os alcoólicos podem apresentar graveis problemas cognitivos que incluem deficiências na memória recente e remota durante semanas ou meses depois do consumo de álcool (SCHUCKIT, 2005)

Os neurônios passam por dois períodos de vulnerabilidade a fatores ambientais durante o desenvolvimento do sistema nervoso central (SHETTY et al., 1993), esses fatores incluem substâncias endógenas, como fatores de crescimento, e xenobióticos (substâncias estranhas ao organismo, como fármacos), dentre estes pode-se citar o álcool (MOONEY \& MILLER, 2007). Nas células, o etanol rompe as montagens dos elementos contráteis do citoesqueleto no músculo cardíaco e na crista neural (MILLER, 1993).

Estudos recentes demonstram que mesmo a exposição pré-natal ao etanol tem sido associada a varias anormalidades em fetos e descendentes. No córtex pré-frontal a exposição aguda ao etanol provoca heterotopia de neurônios da zona sub-ventricular na rota tangencial para o bulbo olfatorio, causando mudanças na desnidade neuronial (FERREIRA et al., 2004).

Síndrome Alcoólica Fetal foi primeiramente descrita por Lemoine em 1968, onde relatou casos de mulheres que beberam durante a gravidez e características das crianças ao nascer (D'ANGIULLI et al., 2006), todavia o termo SAF foi introduzido cinco anos depois por Jones e Smith em 1973, que publicaram uma série de artigos sobre o assunto, tendo uma contribuição valiosa para a determinação da etiologia e caracteristicas da SAF. A SAF é a mais severa manifestação dos efeitos deletéricos do álcool sobre o desenvolvimento fetal (D'ANGIULLI et al., 2006) é uma condição que ocorre somente em crianças de mães que beberam durante a gravidez, os recém nascidos possuem malformação craniofacial, física e mental (RAO \& CHAUDHURI, 2007), deficiência de crescimento pré-natal e pósnatal (KIMPEL et al., 2007), além de redução do volume de uma série de estruturas cerebrais (D'ANGIULLI et al., 2006; CALHOUN et al., 2007).

As crianças que não preenchem todos os critérios exigidos para o diagnóstico de SAF ainda podem mostrar déficit físico e mental compatíveis com um fenótipo parcial conhecido como "Efeitos Fetais do Álcool" (EFA) ou "Distúrbios do desenvolvimento neurológico relacionados com o álcool" (CALHOUN et al., 2007).

Novas técnicas de imagem como Diffusion Tensor Imaging revelaram anormalidades no corpo caloso que estão associadas com redução na atenção e déficits de memória, e a Tomografia de Emissão de Prótons (PET) mostrou um hipometabolismo nos lobos frontais (ALDERAZI \& BRETT, 2007). MENEGOLA et al. (2001), estudaram os mecanismos de teratogênese do álcool, mais precisamente seu metabólito oxidado o acetaldeído, que foi capaz de induzir apoptose (morte celular programada) in vitro (HENGARTNER, 2000). A apoptose é um mecanismo natural do organismo de controle da proliferação celular, que também esta envolvida com a teratogênese observada nos infantes com SAF. Estas exibem déficits no funcionamento cognitivo (D'ANGIULLI et al., 2006), incapacidade na aquisição de informação verbal, compreensão de mundo (RILEY \& MIGEE, 2005), falta de coordenação motora necessária para a performance de movimentos elaborados (OLADEHIN et al., 2007). O Acetaldeído, em recente revisão de SEITZ \& STICKEL (2007), é um importante carcinógeno por interferir com a síntese e reparo de DNA, e causar estresse oxidativo. 
A decisão de ingerir bebidas alcoólicas durante a gravidez trará conseqüências ao longo de toda a vida do concepto, porém, a ação do álcool no feto dependerá do período da gestação, STREISSGUTH et al., (1980), demonstrou um impacto negativo maior no início da gravidez quando comparado com o quinto mês gestacional. A ingestão materna do álcool no primeiro trimestre de gravidez foi associada anomalia craniofacial e a dismorfologia facial também é observada em camundongos expostos ao etanol em estágios equivalentes da gestação (IERACl \& HERRERA, 2007). Segundo CARNEIRO et al. (2005), a exposição do etanol durante o período pré-natal em ratos produz efeitos a longo prazo no desenvolvimento e características farmacológicas do sistema cerebral podendo ter importantes implicações para o desenvolvimento comportamental e neuroquímico na vida adulta do animal.

OLADEHIN et al. (2007), realizaram um estudo com a exposição ao álcool no início da vida pós-natal, focalizando o déficit motor encontrado nos pacientes com SAF, constatando que mesmo a exposição pós-natal ao álcool é capaz de alterar o córtex somatosensorial, o que também ocorre com a exposição pré-natal, seus resultados são similares ao de MARGRET et al. (2005), cuja pesquisa tratou de animais expostos pré-natal ao álcool. Desta forma, neste caso, a exposição ao álcool, sobre diferentes períodos, afeta igualmente o desenvolvimento cerebral.

MOONEY \& MILLER, 2007, avaliaram o efeito do álcool nos períodos críticos de vulnerabilidade: gastrulação e neurogênesis. Foi observado que se a exposição ocorrer durante a gastrulação verifica-se uma condição semelhante a malformação craniofacial encontrada na SAF, enquanto que se ocorrer no período da neurogênesis não ocorre tal condição, os mesmos autores demonstraram que o etanol é neurotóxico (MOONEY \& MILLER, 2003). A gastrulação é o período quando o embrião começa a se organizar em três camadas germinativas, já na neurogênese essas camadas já estão formadas.

A exposição do feto ao álcool é implicada como a causa mais comum e prevenível de retardamento mental nos Estados Unidos (ABEL \& SOKOL, 1991). Este retardo mental reflete as complexas interações do álcool com a organogênese, particularmente a neurogênese. IERACI et al. (2007) demonstrou uma diminuição na neurogênese em camundongos com exposição pós-natal ao álcool. É no girus denteado, parte do hipocampo, que se encontram células tronco/progenitoras capazes de produzir novos neurônios, o que está associado aos processos de memória, aprendizado e humor.

D'ANGIULLI et al. (2006), fez uma revisão de estudos de eletroencefalografia de crianças com SAF, os dado demonstraram que o EEG apresentou-se hipersincrônico refletindo num aumento no número de despertar. Uma noite mal dormida leva a um menor aproveitamento do dia, de certa forma isto pode contribuir para a dificuldade de aprendizado e os problemas comportamentais dos pacientes com SAF.

RAO \& CHAUDHURI (2007), pesquisaram o efeito gestacional do álcool na formação da memória de longo prazo em pintos recém nascidos, o modelo segundo referências dos próprios autores serve tanto para determinação dos efeitos do álcool no início da gravidez, sendo uma poderosa ferramenta para estudo de formação da memória. Este trabalho demonstrou dificuldade na formação da memória na exposição pré-natal ao álcool.

Ao redor de 35\% dos bebedores apresentam as vezes um período de "Ausência", um episodio de amnésia anterógrada temporal, na qual a pessoa esquece parte ou tudo o que aconteceu durante uma seção etílica (SCHUCKIT, 2005). ALDERAZI \& BRETT (2007), em seu trabalho discorrem sobre a ação do álcool no sistema nervoso, dois pontos são focados primeiro os "Blackouts" de memória que podem ocorrer em pessoas muito alcoolizadas ou durante bebidas sociais. Um dos mecanismos é a interferência na ativação do receptor NMDA que possui papel chave no estabelecimento da memória de longa duração. Um efeito indireto do álcool sobre o organismo é a deficiência em tiamina, um importante co-fator para muitas enzimas e não é sintetizado pelo organismo. Durante o consumo de bebidas alcoólicas a ingestão de tiamina é deficiente e o álcool interfere em sua absorção pelo organismo, tornando a deficiência ainda maior.

Muitos estudos, aqui discutidos e outros, demonstram que a exposição pré-natal ao álcool danifica muitas áreas do cérebro que estão envolvidas com a regulação do movimento, atenção, memória entre outros. Os estudos com fetos de animais que foram expostos ao álcool demonstram claramente os efeitos diretos e indiretos do álcool. A falta de informação e apoio psicológico, social leva muitas mulheres a continuar bebendo, mesmo durante a gravidez, sem saber das conseqüências que isto poderá trazer para o bebê.

\section{Alcoolismo e genética.}


Quem não conhece ou nunca ouviu falar de uma família com problemas de alcoolismo? O pai, o tia, a prima, o avô e o filho e os que parentes que morreram por bebida? O alcoolismo é um transtorno complexo influenciado por aspectos genéticos, quase $60 \%$ de sofrer-o depende dos genes (SCHUCKIT, 2005). Segundo KÖHNKE (2008), estudos entre familiares, gêmeos e adoção revelam uma hereditariedade de $50 \%$ no alcoolismo. Baseados nessas questões muitos estudos tentam correlacionar alcoolismo e genética. Com o apoio do National Institute on Alcohol Abuse and Alcoholism teve início em 1989 o Estudo Colaborativo da Genética do Alcoolismo (COGA), que está fazendo uma pesquisa com alcoolistas e seus familiares na iniciativa de identificar as bases genéticas do alcoolismo. Este estudo tem dado muitos frutos como o do gene chrm2 que codifica uma proteína formadora do receptor colinérgico muscarínico M2, onde a variante desse gene fornece um risco aumentado para o alcoolismo (NIAAA). Contudo, também há variantes genéticas protetoras como o ALDH1, gene que codifica a enzima acetaldeídodesidrogenase, onde essa variante, encontrada principalmente em asiáticos, codifica uma forma lenta da enzima o que resulta num acúmulo de acetaldeído que causa desconforto, enjôos e náuseas, o que aumenta o reforço negativo no cérebro diminuindo ou cessando o consumo de álcool (SCHUCKIT, 2005).

HARD et al. (2005), analisou a expressão de genes após a exposição ao álcool e demonstrou que altera o desenvolvimento do camundongo, encontrando alterações em genes como o Bmp15, membro da superfamília de TGF-B e Rnf25 um facilitador da ubiquitinação, um processo pelo qual as proteínas são marcadas para proteólise. KIMPEL et al. (2007), analisaram o perfil da expressão de genes em populações de ratos que preferiam álcool e os que não preferiam, este critério foi baseado em trabalhos de outros autores, onde os ratos tinham livre acesso a etanol $10 \%(V / N)$ e água, os ratos que preferiam álcool possuem dependência física e consumiam $5 \mathrm{~g} / \mathrm{Kg} / \mathrm{dia}$, enquanto os que não preferiam consumiam menos que $1 \mathrm{~g} / \mathrm{Kg} / \mathrm{dia}$. A expressão de genes foi realizada em cinco regiões cerebrais núcleo acumbens, amígdala, córtex frontal, putamem-caudado e hipocampo. $\mathrm{O}$ trabalho analisa todos os genes e suas alterações de região a região. Por exemplo, região da amígdala foi a que mostrou maior diferença no perfil de expressão, seguida pela do caudado-putamen, hipotálamo, acumbens e córtex frontal, sugerindo uma maior participação da amígdala no alcoolismo.

Pesquisas sobre a genética do alcoolismo são fundamentais não só para o entendimento da patogênese, bem como possibilitar de maneira sólida conhecimento para o indivíduo sobre os riscos para sua saúde (NORNBERGER \& BIERUT, 2007). O alcoolismo é uma doença que tem crescido mundialmente, a busca por novos tratamentos e alvos farmacológicos certamente será ajudada com o desenvolvimento das bases genéticas do alcoolismo.

\section{Tratamento farmacológico do alcoolismo}

Hoje existem nos EUA três fármacos aprovados para o tratamento do alcoolismo: dissulfiram, naltrexona $e$ acamprosato. O dissulfiram tem sido usado há muito tempo, mas caiu em descrédito por seus efeitos colaterais e pelos problemas de adesão ao tratamento. A naltrexona e o acamprosato foram introduzidos mais recentemente. $\mathrm{O}$ objetivo desses fármacos é ajudar o paciente a manter a abstinência (FLEMING et al., 2006).

A naltrexona $\left(\operatorname{ReVia}{ }^{\circledR}\right.$, nome pelo qual é vendido nos EUA) foi aprovada pelo FDA para o tratamento do alcoolismo em 1994 (O'MALLEY et al., 1992; VOLPICELLI et al., 1992). Esse fármaco esta relacionada quimicamente com o antagonista altamente seletivo dos receptores opióides conhecido como naloxona, mas sua biodisponibilidade oral e a duração da ação são maiores. A naltrexona é um antagonista opiáceo. Sabe-se, há mais de duas décadas, que o álcool altera a atividade dos receptores opiáceos (JOHNSON \& AIT-DAOUD, 2000; LEE et al., 2005). Aparentemente, o álcool aumenta a atividade desses receptores; o efeito desta estimulação aguda estaria envolvido na sensação de euforia produzida pelo álcool, que é um dos efeitos mais reforçadores desta droga ( DE WAELE et al, 1992).

O consumo de álcool aumenta a liberação de $\beta$-endorfinas em regiões cerebrais como o nucleo accumbens (MARINELLI et al., 2004), um efeito que está bloqueado pela naltrexona (ZALEWSKA-KASZUBSKA et al., 2006). Sabe-se que ratos criados para ter uma preferência por álcool mostram um aumento na liberação de $\beta$-endorfinas quando comparados com ratos sem preferência por álcool (deWAELE et al, 1992; ZALEWSKA-KASZUBSKA et al., 2006). Em humanos, (GIANOULAKIS, 1993) mostrou que homens bebedores sociais tinham um risco aumentado para abuso de álcool, pois possuíam pais com problemas relacionados com o álcool, estes pacientes apresentaram um aumento de $170 \%$ nos níveis de $\beta$-endorfinas periféricas após consumirem uma dose moderada de álcool quando comparados com controles. 
JOHNSON (2008), sugere que as diferenças genéticas moleculares que alteram a expressão de $\beta$-endorfinas, não apenas os seus níveis plasmáticos, modulam o nível de resposta a naltrexona. No entanto, existe uma crescente evidência em humanos que as diferenças nos genes de receptores opióides mu OPRM1 estão associados com a resposta terapêutica a naltrexona (McGEARY et al., 2006; OSLIN et al., 2003). Recentemente, foi proposto que os indivíduos com variante Asp do gene OPRM1 exibiram preferencialmente uma alta taxa de prevenção de recaídas quando receberam tratamento com naltrexona (OSLIN et al., 2003).

Estudos com animais e a experiência clinica sugeriram que a naltrexona pode reduzir o consumo e o desejo de ingerir álcool; isso foi confirmado por estudos clínicos (O'MALLEY et al., 2000; JOHNSON \& AIT-DAOUD, 2000). Existem evidências de que a naltrexona bloqueia a ativação das vias dopaminérgicas cerebrais pelo álcool, que parecem ser fundamentais para a sensação de recompensa (VASCONCELOS et al., 2001).

VOLPICELLI et al. (1992) organizaram um estudo para avaliar a eficácia do naltrexona em reduzir as taxas de recaída nesses pacientes. Por 12 semanas, em um estudo duplo-cego, administraram 50mg de naltrexona ou placebo em 70 pacientes. Além da medicação, esses pacientes recebiam aconselhamento em relação ao alcoolismo, terapia de prevenção de recaídas e encaminhamento para Alcoólicos Anônimos. Houve uma redução substancial no desejo para beber ao longo das 12 semanas no grupo que recebeu naltrexona. Consequentemente houve também uma diminuição no consumo de álcool do grupo experimental. Quando os pacientes do grupo placebo bebiam, eles o faziam em quantidades quatro vezes superiores ao grupo experimental.

O'MALLEY et al (1992), também administraram 50mg de naltrexona ou placebo por 12 semanas para 97 pacientes e da mesma forma encontraram no grupo experimental uma redução do consumo de álcool e menor número de episódios de recaídas. Foram desenvolvidas três formulações de liberação prolongada de naltrexona para injeção intramuscular profunda - Vivitrol ${ }^{\circledR}$, Natrel ${ }^{\circledR}$ e Depotrex $^{\circledR}$ (JOHNSON, 2008).

O nalmefeno é outro antagonista opióide que tem efeitos aparentemente promissores nos testes clínicos preliminares. Esse fármaco apresenta algumas vantagens sobre a naltrexona, incluindo-se a biodisponibilidade oral maior, a duração de ação mais longa e a inexistência de hepatotoxicidade dependente da dose (MASON et al., 1999).

O dissulfiram foi usado durante uma pesquisa sobre sua eficácia anti-helmíntica potencial por dos médicos dinamarqueses. Eles iniciaram uma série de estudos clínicos e farmacológicos, constituindo a base para a utilização do dissulfiram como coadjuvante no tratamento de alcoolismo crônico (FLEMING et al., 2006). Administrado isoladamente, o dissulfiram é uma substância relativamente atóxica, mas inibe a atividade da ALDH (Aldeído Desidrogenase) e aumenta a concentração sanguínea de acetaldeído 5 a 10 vezes acima do nível alcançado quando o etanol é administrado a um individuo que não foi tratado com dissulfiram. Depois da administração de dissulfiram, as isoformas citosólicas e mitocondrial da ALDH são inativadas reversivelmente em graus variáveis e a concentração de acetaldeído aumenta (JOHANSSON, 1992; JOHNSON \& AIT-DAOUD, 2000). O dissulfiram e seus metabólitos podem inibir algumas enzimas com grupos sulfidrílicos essências e, desse modo, produzem grandes variedades de efeitos biológicos. Esse fármaco inibe as CYP hepáticas e, por esse motivo, interfere no metabolismo da fenitoína, do clordiazepóxido, dos barbitúricos, da warfarina e de outros fármacos. Em geral, o próprio dissulfiram é inócuo, mas pode causar erupções acneiformes, urticária, lassidão, tremor, agitação, cefaléia, tontura, gosto de alho ou de metal e distúrbios gastrintestinais suaves. Também existem relatos de neuropatias periféricas, psicose e cetose (FLEMING et al., 2006; O’SHEA, 2000).

O acamprosato é um análogo do GABA (LITTEN et al., 1996) e também análoga ao Ácido L-Glutâmico (KNÖPFEL et al., 1987) amplamente utilizado na Europa para o tratamento do alcoolismo. Recentemente, foi aprovado nos EUA e esta sendo introduzida no mercado brasileiro pela Merck. Alguns estudos duplo-cegos controlados por placebo mostraram que o acamprosato reduz a freqüência da ingestão de álcool e as recaídas em alcoólicos abstêmios. A ação desse fármaco é dependente da dose (1,3 a 2 g/dia) (PAILLE et al., 1995).

Pesquisas com animais de laboratório mostraram que o acamprosato reduz a ingestão de álcool sem afetar o consumo de água e alimentos (CZACHOWSKI, 2001). Em geral, esse fármaco é bem tolerado pelos pacientes e seu principal efeito colateral é a diarréia (GARBUTT et al., 1999). Nenhum estudo mostrou potencial de uso abusivo. O fármaco sofre metabolismo mínimo no fígado, é excretado principalmente pelos rins e tem meia-vida de eliminação de $18 \mathrm{~h}$ após a administração oral (WILDE \& WAGSTAFF, 1997). O uso simultâneo de dissulfiram parece acentuar a eficácia do acamprosato, sem quaisquer interações farmacológicas adversas detectáveis (BESSON et al., 1998). 
O principal efeito neuroquímico de Acamprosato foi atribuída ao antagonismo dos receptores de glutamato NMDA (De WITTE et al., 2005; JOHNSON \& AIT-DAOUD, 2000). Recentemente foi proposto que acamprosato modula a neurotransmissão glutamatérgica em receptores 5-metabotrópico (mGluR5) (HARRIS et al., 2002).

O Acamprosato demonstrou diminuição: (a) Consumo de etanol em roedores (BOISMARE et al., 1984; LE MAGNEN, 1987; CZACHOWSKI, 2001), (b) Hiperexcitabilidade de dopamina no núcleo accumbens durante abstinência alcoólica (DAHCHOUR et al., 1998; ROSSETTI \& CARBONI, 1995), (c) Hiperexcitabilidade neuronal geral (GEWISS et al., 1991; SPANAGEL et al., 1996), (d) Neutrotransmissão glutamatérgica em camundongos dependentes de álcool (DAHCHOUR et al., 1998; BOLO et al., 1998), (e) A atividade dos canais de cálcio voltagem dependente e a expressão do gene c-fos do cérebro associado com a retirada de álcool (PUTZKE et al., 1996).

O topiramato é usado no tratamento dos distúrbios convulsivos e parece ser útil também para tratar a dependência alcoólica (JOHNSON, 2008). Em comparação com o grupo placebo, os pacientes que usaram topiramato conseguiram maior número de dias em abstinência e sentiram menos desejo irrefreável de ingerir álcool (JOHNSON et al., 2003; FLEMING et al., 2006). Topiramato é um derivado da D-frutose (2,3:4,5-bis-O-(1metiletiledeno)-ß-frutopiranose sulfamato, monosacarídeo comum que contém grupo sulfatado, tem seis importantes mecanismo de ação adicional a sua capacidade de antagonizar os receptores do ácido I - amino - 3 - hidroxi - 5 metilisoxasol - 4 - propiónico e receptores glutamato tipo kainato (GIBBS et al., 2000; GRYDER \& ROGAWSKI, 2003);

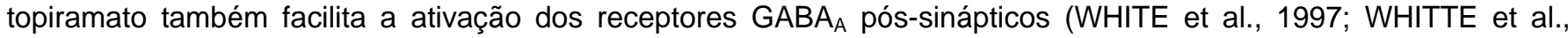
2000); inibe os canais de cálcio tipo-L e o sistema de segundos mensageiros cálcio dependentes (ZHANG et al., 2000); reduz a atividade dos canais de sódio de lata voltagem (TAVERNA et al., 1999); ativa a condutância de potássio (HERRERO et al., 2002); é um fraco inibidor das isoenzimas da anidrasa carbônica CA-II e CA-IV (DODGSON et al., 2000) ambas são encontrados em tecidos periféricos e neuronal. Em túbulos renais a inibição da anidrasa carbônica reduz a secreção de iones de hidrogênio e aumenta a secreção de $\mathrm{Na}^{+}, \mathrm{K}^{+}, \mathrm{HCO}_{3}^{-}$e água aumentando assim a probabilidade de acidose renal e formação de pedras (DODGSON et al., 2000; SHANK et al., 2000).

Durante três décadas, tem havido intenso interesse em os efeitos dos agentes serotoninérgicos no tratamento da dependência do álcool, incentivado pelo aumento do conhecimento sobre vários tipos de receptores 5-HT (JOHNSON, 2008). A ondansetrona, um antagonista dos receptores 5- $\mathrm{HT}_{3}$ e antiemético reduz o consumo de álcool em animais de laboratório (BOYCE-RUSTAY et al., 2006) e, hoje, esta sendo testado em seres humanos. Os resultados preliminares sugerem que a ondansetrona é eficaz para o tratamento do alcoolismo de início precoce, que responde mal às terapias psicossociais aplicadas isoladamente (JOHNSON, 1993). Contudo, esse fármaco não parece atuar bem nos outros tipos de alcoolismo (JOHNSON \& AIT-DAOUD, 2000). A administração de ondansetrona reduz quantidade de álcool consumida, principalmente por alcoólicos que ingerem menos de 10 drinques por dia (SELLERS et al., 1994).

No entanto, a combinação terapêutica de naltrexona e acamprosato foi proposta a ser de potencial terapêutico pelos seguintes razões: Naltrexona reduz os efeitos de reforço do álcool (KOOB, 1992) e acamprosato modula a abstinência alcoólica induzida por aumento de glutamato extracelular no sistema cortico-mesolimbico (DAHCHOUR, 2003) e acamprosato aumenta os níveis sanguíneos de naltrexona, aumentando assim seus efeitos neuroquímicos (JOHNSON et al., 2003).

\section{CONSIDERAÇÕES FINAIS}

O etanol é uma droga milenar e muito consumida nos dias atuais. É de fácil obtenção, encontrada em prateleiras de supermercados, padarias, entre outros. Embora seja uma droga estudada por vários pesquisadores em todo o mundo, aspectos importantes, como por exemplo, o mecanismo de ação ainda não está totalmente elucidado. Diversos estudos demonstram o impacto negativo do álcool sobre vários órgãos e sistemas, principalmente sobre o desenvolvimento e formação do SNC. A Síndrome Alcoólica Fetal é uma prova bem clara deste efeito deletério. O álcool afeta vários, se não todos, sistemas de neurotransmissores do SNC. Os estudos com álcool precisam avançar e novos modelos experimentais (comportamento, dosagem de receptores, dosagem de neurotransmissores, entre outros), mais específicos, devem ser desenvolvidos. Com o surgimento de ferramentas que possibilitam o escaneamento do genoma de indivíduos com forte tendência ao alcoolismo ou através de modelos animais, espera-se entender os mecanismos moleculares do desenvolvimento e instalação do alcoolismo, esse conhecimento trará novos 
alvos para o tratamento farmacológico, bem como uma melhor decisão de escolha para o tratamento dos indivíduos mais susceptíveis.

\section{Apoio financeiro- CNPq, CAPES e FUNCAP}

\section{REFERÊNCIAS BIBLIOGRAFICAS}

ABEL, E. L.; SOKOL, R. J. A revised conservation estimate of the incidence of FAS and its economic impact. Alcoholism: Clinical and Experimental Research. v. 15, p. 514-524, 1991.

AGUAYO, L. G.; PEOPLES R. W.; YEH H. H.; YEVENES G. E. GABA(A) receptors as molecular sites of ethanol action. Direct or indirect actions? Current Topics in Medicinal Chemistry. v. 2, p. 869-885, 2002.

ALDERAZI, Y.; BRETT, F. Alcohol and the nervous system. Currrent Diagnostic Pathology. v.13, p.203-2006, 2007.

ARAUJO, V. R. K.; RIBEIRO, M. M.; SILVA, A. J. L.; ALBUQUERQUE, M. L.; TELES, P. N. F. Alterações morfométricas no Timo, Baço e Placas de Peyer durante a exposição pré e pós-natal ao álcool. Revista Eletrônica de Farmácia. v.4, n.1, p.32-42, 2007.

BARNES, N. M.; SHARP, T. A review of central 5-HT receptors and their function. Neuropharmacology. v.38, p.1083$1152,1998$.

BESSON, J.; AEBY, F.; Kasas, A.; Lehert, P.; Potgieter, A. Combined efficacy of acamprosate and dissulfiram in the treatment of alcoholism: A controlled study. Alcoholism: Clinical and Experimental Research. v.22, p.573-579, 1998.

BOISMARE, F.; DAOUST, M.; MOORE, N.; SALIGAUT, C.; LHUINTRE, J. P.; CHRETIEN, P.; DURLACH, J. A homotaurine derivative reduces the voluntary intake of ethanol by rats: are cerebral GABA receptors involved? Pharmacology Biochemistry \& Behavior. v.21, p.787-789, 1984.

BOLO, N.; NÉDELEC, J. F.; MUZET, M.; DE WITTE, P.; DAHCHOUR, A.; DURBIN, P.; MACHER, J. P. Central effects of acamprosate. Part 2. Acamprosate modifies the brain in-vivo proton magnetic resonance spectrum in healthy young male volunteers. Psychiatry Research: Neuroimaging. v.82, p.115-1127, 1998.

BOYCE-RUSTAY, J. M.; WIEDHOLZ, L. M.; MILLSTEIN, R. A.; CARROLL, J.; MURPHY, D.L.; DAWS, L. C.; HOLMES, A. Ethanol-related behaviors in serotonin transporter knockout mice. Alcoholism: Clinical and Experimental Research. v.30, p.1957-65, 2006.

BROOKS, P. J. Brain atrophy and neuronal loss in alcoholism: a role for DNA damage? Neurochemistry International. v. 37, p.403-412, 2000.

CALHOUN, F.; KENNETH, W. Fetal alcohol syndrome: Historical perspectives. Neuroscience and Biobehavioral Reviews. v. 31, p.168-171, 2007.

CARBONI, E.; ACQUAS, E.; FRAU, R.; DI CHIARA, G. Differential inhibitory effects of a 5-HT3 antagonist on druginduced stimulation of dopamine release. European Journal of Pharmacology. v. 164, p. 515-9, 1989.

CARNEIRO, L. M., DIÓgENES, J. P.; VASCONCELOS, S. M.; ARAGÃO, G. F.; NORONHA, E. C.; GOMES, P. B.; VIANA, G. S. B. Behavioral and neurochemical effects on rat offspring after prenatal exposure to ethanol. Neurotoxicology and Teratology. v.27, p. 585-592, 2005.

CEBRID- Centro Brasileiro de Informações sobre o uso de drogas. II Levantamento Domiciliar sobre uso de drogas psicotropicas no Brasil. Departamento de Psicobiologia, UNIFESP, 2005.

CZACHOWSKI, C. L.; LEGG, B. H.; SAMSON, H. H. Effects of acamprosate on ethanol-seeking and selfadministration in the rat. Alcoholism: Clinical and Experimental Research. v.25, p.344-50, 2001. 
DAHCHOUR, A.; DE WITTE, P.; BOLO, N.; NEDELEC, J. F.; MUZET, M.; DURBIN, P. ; MACHER JP. Central effects of acamprosate. Part 1. Acamprosate blocks the glutamate increase in the nucleus accumbens microdialysate in ethanol withdrawn rats. Psychiatry Research: Neuroimaging. v.82, p.107-114, 1998.

DAHCHOUR, A.; DE WITTE, P. Effects of acamprosate on excitatory amino acids during multiple ethanol withdrawal periods. Alcoholism: Clinical and Experimental Research. v.27, p.465-70, 2003.

DE WAELE, J.P.; PAPACHRISTOU, D.N.; GIANOULAKIS, C. The alcohol-preferring C57BL/6 mice present an enhanced sensitivity of the hypothalamic B-endorphin system to ethanol than the alcohol-avoiding DBA/2 mice. Journal of Pharmacology and Experimental Therapeutic. v.261, p.788-794, 1992.

De WITTE, P.; BACHTELER, D.; SPANAGEL, R. Acamprosate preclinical data. In: SPANAGEL R. Drugs for relapse prevention of alcoholism. Basel, Switzerland. Verlag, 2005.

DILDY-MAYFIELD, J. E.; HARRIS, R. A. Neurobiology of alcohol's actions and the addictive process. In: TABAKOFF B.; HOFMANN P.L, ed. Biological Aspect of Alcoholism. Seattle, Hogrefe \& Huber Publishers. p.189-224, 1995.

DODGSON, S. J.; SHANK, R. P.; MARYANOFF, B. E. Topiramate as an inhibitor of carbonic anhydrase isoenzymes. Epilepsia. v.41(suppl 1), p.S35-39, 2000.

D'ANGIULLI, A.; GRUNAU, P.; MAGGI, S.; HERDMAN, A. Electroencephalographic correlates of prenatal exposure to alcohol in infants and children: a review of findings and implications for neurocognitive development. Alcohol. p.127133, 2006.

DURAZZO, T. C.; GAZDZINSKI, S.; MEYERHOFF, D. J. The Neurobiological and Neurocognitive Consequences of Chronic Cigarette Smoking in Alcohol use disorders. Alcohol \& Alcoholism. v.42, n.3, p.174-185, 2007.

FERNANDES, N.; FERNANDES, V. Criminologia Integrada. Revista dos Tribunais. 2 ed. São Paulo. Toxicomanias, p.679-729, 2002.

FERREIRA, V. M. M.; MORATO, G. S. D-Cycloserine blocks the effects of ethanol and HA- 906 in rats tested in the elevated plus-maze. Alcoholism: Clinical and Experimental Research. v.1, n.9, p. 1638-1642, 1997.

FERREIRA, T. A. A.; MORAIS, J. R.; FERREIRA, N. R.; PENHA-SILVA, N. Effects of acute prenatal exposure to ethanol on the postnatal morphology of the prefrontal córtex in wistar rats. Brazilian Journal of Morphological Sciences. v.21, n.2, p.97-101, 2004.

FLEMING, M.; MIHIC, S. J.; HARRIS, R. A. Etanol. In: Lazo J.S.; PARKER K.L. Goodman \& Gilman: As Bases Farmacológicas da Terapêutica. São Paulo: Editora Mac GrawHill, 2006.

FRIEND, K. B.; MALLOY, P. F.; SINDELAR, H. A. The effects of chronic nicotine and alcohol use on neurocognitive function. Addictive Behaviors. v. 30, p.193-202, 2005.

GARBUTT, J. C.; WEST, S. L.; CAREY T.S. et al. Pharmacological treatment of alcohol dependence: A review of the evidence. Journal of the American Medical Association. v.281, p. 1318-1325, 1999.

GAZAL-CARVALHO, C.; CARLINI-COTRIM, B.; SILVA, O. A.; SAUAIA, N. Prevalência de alcoolemia em vítimas de causas externas admitidas em centro urbano de atenção ao trauma. Revista de Saúde Pública. v. 36, n.1, p. 47-54, 2002.

GEWISS, M.; HEIDBREDER, C.; OPSOMER, L.; DURBIN, P.; DE WITTE, P. Acamprosate and diazepam differentially modulate alcohol-induced behavioural and cortical alterations in rats following chronic inhalation of ethanol vapour. Alcohol Alcohol. v.26, p.129-137, 1991.

GIANOULAKIS, C. Endogenous opioids and excessive alcohol consumption. Journal of Psychiatric Neuroscience. v.18, n.4, p.148-156, 1993. 
GIANOULAKIS, C. Implications of endogenous opióides and dopamine in alcoholism: human and basic science studies. Alcohol. v.1, p.33-42, 1996.

GIANOULAKIS, C. Alcohol-seeking behavior. The roles of the hypothalamic-pituitary-adrenal axis and the endogenous opioid system. Alcohol Health \& Research World. v.22, p.202-10, 1998.

GIBBS, J. W.; SOMBATI, S.; DELORENZO, R. J.; COULTER, D. A. Cellular actions of topiramate: blockade of kainateevoked inward currents in cultured hippocampal neurons. Epilepsia. v.41(suppl 1), p.S10-16, 2000.

GORDON, A.S.; DIAMOND, I. Adenosine mediates the effects of ethanol on the CAMP signal transduction system. In: TABERNER PV, BADAWY AAB, editors. Advances in Biomedical Alcoholism Research. Oxford: Pergamon Press; 1993.

GRYDER, D. S.; ROGAWSKI, M. A. Selective antagonism of GluR5 kainate-receptor-mediated synaptic currents by topiramate in rat basolateral amygdala neurons. Journal of Neuroscience. v.23, p.7069-7074, 2003.

GROBIN, A. C.; MATTHEWS, D. B.; DEVAUD, L. L.; MORROW, A. L. The role of GABA(A) receptors in the acute and chronic effects of ethanol. Psychopharmacology. v.139, p. 2-19, 1998.

HARRIS, B. R.; PRENDERGAST, M. A.; GIBSON, D. A.; ROGERS, D. T.; BLANCHARD, J. A.; HOLLEY, R. C.; FU, M.C.; HART, SR.; PEDIGO, NW, LITTLETON, JM. Acamprosate inhibits the binding and neurotoxic effects of transACPD, suggesting a novel site of action at metabotropic glutamate receptors. Alcoholism: Clinical and Experimental Research. v.26, p.1779-93, 2002.

HARD M. L.; ABDOLEL M.; ROBINSON B. H.; KOREN G. Gene-expression analysis after alcohol exposure in the developing mouse. Journal of Laboratory and Clinical Medicine. v. 145, n.1, p. 47-54, 2005.

HENGARTNER, M. O. The biochemistry of apoptosis. Nature. v. 407, p. 770-776, 2000.

HERRERO, A. I.; DEL OLMO, N.; GONZALEZ-ESCALADA, J. R.; SOLIS, J. M. Two new actions of topiramate: inhibition of depolarizing GABA(A)-mediated responses and activation of a potassium conductance. Neuropharmacology v.42, p.210-20, 2002.

HINGSON, R.; WINTER, M. Epidemiology and Consequences of Drinking and Driving. Alcohol Research \& Health. v.7, n.1, p.63-73, 2003.

IERACI, A.; HERRERA, D. G. Single alcohol exposure in early life damages hippocampal stem/progenitor cells and reduces adult neurogenesis. Neurobiology of Disease. v. 26, p. 597-605, 2007.

JOHANSSON, B. A review of the pharmacokinetics and pharmacodynamics of dissulfiram and its metabolites. Acta Psychiatrica Scandinavica. Supplementum. v.369, p.15-26, 1992.

JOHNSON, B.A.; AIT-DAOUD, N. Neuropharmacological treatments for alcoholism: Scientific basis and clinical findings. Psychopharmacology. v.149, p. 327-344, 2000.

JOHNSON, B. A. ; AIT-DAOUD, N. ; BOWDEN, C. L. ; DICLEMENTE, C. C. ; ROACHE, J. D. ; LAWSON, K. ; JAVORS, M. A. ; MA, J. Z. Oral topiramate for treatment of alcohol dependence: A randomized, controlled trial. Lancet. v.361, p.1677-1685, 2003.

JOHNSON, B. A. Update on neuropharmacological treatments for alcoholism: Scientific basis and clinical findings. Biochemical Pharmacology. v.75, p.34-56, 2008.

JOHNSON, B. A.; CAMPLING, G. M.; GRIFFITHS, P.; COWEN, P. J. Attenuation of some alcohol-induced mood changes and the desire to drink by 5-HT3 receptor blockade: a preliminary study in healthy male volunteers. Psychopharmacology. v.112, p.142-4, 1993. 
JOHNSON, B. A.; O'MALlEY, S. S.; CIRAULO, D. A.; ROACHE, J. D.; CHAMBERS, R. A.; SARID-SEGAL, O,; COUPER, D. Dose-ranging kinetics and behavioral pharmacology of naltrexone and acamprosate, both alone and combined, in alcoholdependent subjects. Journal of Clinical Psychopharmacology. v.23, p.281-93, 2003.

KATZUNG, B. Farmacologia Básica e Clínica. 9a edição. Editora Guanabara Koogan. 2006.

KENNEDY, R.H.; LIU, S.J. Sex differences in L-type calcium current after chronic ethanol consumption in rats. Toxicology and Applied Pharmacology. v.15, n.3, p.196-203, 2003.

KIMPEL, M. W.; STROTHER, W. N.; MCCLINTICK, J. N.; CARR, L. G.; LIANG, T.; EDENBERG, H. J.; MCBRIDE, W. Functional gene expression differences between inbred alcohol-preferring and non-preferring rats in five brain regions. Alcohol. v. 41, p.95-132, 2007.

KNÖPFEL, T.; ZEISE, M. L.; CUÉNOD, M.; ZIEGLGÄNSBERGER, W. L-Homocisteric acid but receptor prefering agonista in rat neocortical neurons in vitro. Neurocience Lett. v.81, p.188-92, 1987.

KÖHNKE, M. D. Approach to the genetics of alcoholism: A review based on pathophysiology. Biochemical Pharmacology. v.75, p. 160-177, 2008.

KOOB, G. F. Neural mechanisms of drug reinforcement. Annals of the New York Academy of Sciences. v.654, p.171191, 1992.

LEE, Y. K.; PARK, S. W.; KIM, Y. K.; KIM, D. J.; JEONG, J.; MYRICK, H.; KIM, Y. H. Effects of naltrexone on the ethanol-induced changes in the rat central dopaminergic system. Alcohol. v.40, p.297-301, 2005.

LE MAGNEN, J.; TRAN, G.; DURLACH, J. Lack of effects of Ca-acetyl homotaurinate on chronic and acute toxicities of ethanol in rats. Alcohol. v.4, p.103-8, 1987.

LITTEN, R. Z.; ALLEN, J.; FERTIG, J. Pharmacoterapies for alcohol problems; A review of research with focus on developments since 1991. Clinical and Alcoholism Experimental Research. v.20, n.5, p.859-76, 1996.

LONGO, L. P.; CAMPBELL, T.; HUBATCH, S. Divalproex sodium (Depakote) for alcohol withdrawal and relapse prevention. Journal of Addictive Diseases. v.21, n.2, p. 55-64, 2002.

MARINELLI, P. W, QUIRION, R.; GIANOULAKIS, C. An in vivo profile of beta-endorphin release in the arcuate nucleus and nucleus accumbens following exposure to stress or alcohol. Neuroscience. v.127, p.777-784. 2004.

MARIN, L.; QUEIROZ, M. S. A atualidade dos acidentes de trânsito na era da velocidade: uma visão geral. Caderno de Saúde Pública. v.16, n.1, p. 7-21, 2000.

MARGRET, C. P.; LI CHENG, X.; ElBERGER, A. J.; CHAPPELL, T. D.; WATERS, R. S. Prenatal alcohol exposure alters the size, but not the pattern, of the whisker representation in neonatal rat barrel cortex. Experimental Brain Research, v.65, p. 167-178, 2005.

MASON, B. J.; SALVATO, F. R.; WILLIAMS, L.D.; RITVO, E. C.; CUTLER, R. B. A double-blind, placebo -controlled study of oral nalmefene for alcohol dependence. Archives of General Psychiatry. v.56, p.719-724, 1999.

MATSUZAWA, S.; SUZUKI, T.; MISAWA, M.; NEGASE, H. Roles of 5-HT3 and opioid receptors in the ethanol-induced place preference in rats exposed to conditioned fear stress. Life Sciences. v.64, p.241-9, 1999.

MENEGOLA, E.; BROCCIA, M. L.; RENZO, F. D.; GIAVINI, E. Acetaldehyde in vitro exposure and apoptosis: a possible mechanism of teratogenesis. Alcohol. v.23, p.35-39, 2001.

MELCHIOR, M.; CHASTANG, J.; GOLDBERG, P.; FOMBONNE, E. High prevalence rates of tobacco, alcohol, and drug use in adolescents and young adults in France: Results from the GAZEL youth study. Addictive Behaviors. v.33, p.122-133, 2008. 
McGEARY, J. E.; MONTI, P. M.; ROHSENOW, D. J.; TIDEY, J.; SWIFT, R.; MIRANDA, R. J. Genetic moderators of naltrexone's effects on alcohol cue reactivity. Alcoholism: Clinical and Experimental Research. v.30, p.1288-96, 2006.

MIHIC, S. J.; HARRIS, R. A. GABA and the GABAA receptor. Alcohol Health Res. World. v.21, n.2, p.127-131, 1997.

MILLER, M. W. Generation of neurons in the rat dentate gyrus and hippocampus: effects of prenatal and postnatal treatment with ethanol. Alcoholism: Clinical and Experimental Research.. v.19, p.1500-1509, 1995.

MILLER, M. W. Migration of cortical neurons in altered by gestation exposure to ethanol. Alcoholism: Clinical and Experimental Research.. v.17, n.2, p.304-314, 1993.

MILLER, M. W. Development of the Central Nervous System: Effects of Alcohol and opiates. New York: Wiley-Liss, 1992.

MINAYO, M. C. S; DESLANDES S.F. A complexidade das relações entre drogas, álcool e violência. Caderno de Saúde Pública. v.14, n.1, p. 35-42, 1998.

MOONEY, S. M.; MILLER, M. W. Time-specific effects of ethanol exposure on cranial nerve nuclei: Gastrulation and Neurogenesis. Experimental Neurology. v. 205, p. 56-63, 2007.

MOONEY S. M.; MILLER, M. W. Ethanol-induced neuronal death in organotipic cultures of rat cerebral cortex. Developmental Brain Research, 147, p. 135-141, 2003.

MOONEY, S. M.; MILLER M. W. Nerve growth factor neuroprotection of ethanol death in rat cerebral cortex is age dependent. Neuroscience, 149, p. 372-381, 2007.

MOONEY, S. M. Stereological study of the effects of postnatal exposure to ethanol on the numbers of neurons in forebrain structures. 1997. Tese (Doutorado). University of Otago. Dunedin, New Zealand.

MYERS, R. D.; VEALE, W. L. Alcohol preference in the rat: reduction following depletion of brain serotonin. Science v.160, p.1469-1471, 1968.

NIAAA - National Institute on Alcohol Abuse and Alcoholism. U.S. Department of Health and Humam Services. $10^{\text {th }}$. Special report to the U.S. Congress on alcohol an health, Bethesda, 2000.

NORNBERGER, J. I.; BIERUT, J. L. Gene e alcoolismo, uma intima interelação. Scientific American Brasil. Ed. 60, 2007.

OLADEHIN, A.; MARGRET, C. P.; MAIER, S. E.; LI CHENG, X.; JAN, T. A.; CHAPPELL, T. D.; WATERS, R. S. Early postnatal alcohol exposure reduced the size of vibrissal barrel field in rat somatosensory cortex (SI) but did not disrupt barrel field organization. Alcohol. v. 41, p. 253-261, 2007

O'MALLEY, S. S.; JAFFE, A. J.; CHANG, G.; SCHOTTENFELD, R. S.; MEYER, R. E.; ROUNSAVILLE, B. Naltrexone and coping skills therapy for alcohol dependence. A controlled study. Archives of General Psychiatry. v.49, n.11, p.881-887, 1992.

O'MALLEY, S. S.; KRISHNAN-SARIN, S.; FARREN, C.; O'CONNOR, P. G. Naltrexone-induced nausea in patients treated for alcohol dependence: Clinical predictors and evidence for opioid-mediated effects. Journal of Clinical Psychopharmacology. v.20, p.69-76, 2000.

OSCAR-BERMAN, M. Neuropsychological vulnerabilities in chronic alcoholism. 2000. 437-472p. NIAAA research monograph No. 34. Review of NIAAA's Neuroscience and Behavioral Research Portfolio, NIAAA, Bethesda.

Oslin, D. W.; Berrettini, W.; Kranzler, H. R.; Pettinati, H.; Gelernter, J.; Volpicelli, J. R.; O'Brien, C. P. A functional polymorphism of the mu-opioid receptor gene is associated with naltrexone response in alcohol-dependent patients. Neuropsychopharmacology. v.28, p.1546-525, 2003.

O'SHEA, B. Disulfiram revisited. Hosp Méd. v.61, p.849-851, 2000. 
PAILLE, F.M. et al. Double-blind, randomized multicentre trial of acamprosate in maintaining abstinence from alcohol. Alcohol. v.30, p.239-247, 1995.

PRANT, G.; ADAN, A.; PÈREZ-PÀMIES, M.; SÀNCHEZ-TURET, M. Neurocognitive effects of alcohol hangover. Addictive Behaviors. v.33, p.15-23, 2008.

PUTZKE, J.; SPANAGEL, R.; TOLLE, T. R.; ZIEGLGANSBERGER, W. The anti-craving drug acamprosate reduces cfos expression in rats undergoing ethanol withdrawal. European Journal of Pharmacology. v.317, p.39-48, 1996.

RANG, H.P; DALE,M.M; RITTER, J.M. Farmacologia. Rio de Janeiro, Editora Elsevier. 2007.

RABBANI, M, TABAKOFF, B. Chronic ethanol treatment reduces adenylyl cyclase activity in human erythroleukemia cells. European Journal of Pharmacology. v.26, p.19- 23, 2001.

RAO, V.; CHAUDHURI, J. D. Effect of gestational ethanol exposure on long-term memory formation in newborn chicks. Alcohol. article in press. v. 41, p. 1-7, 2007.

RILEY, E. P.; MCGEE, C. L. Fetal alcohol spectrum disorders: an overview with emphasis on changes in brain and behavior. Experimental Biology and Medicine. v.230, p.357-365, 2005.

ROSSETTI, Z. L.; CARBONI, S. Ethanol withdrawal is associated with increased extracellular glutamate in the rat striatum. European Journal of Pharmacology. v.283, p.177-83, 1995.

SASAKI, H.; MATSUZAKI, T.; NAKAGAWA, H. A.; SEKIZAWA, K.; MARUYAMA, Y. Cognitive function in rats with alcohol ingestion. Pharmacology Biochemistry Behavior. v.52, n.4, p.845-8, 1995.

SCHAMBRA, U. B.; LAUDER, J. M.; PETRUSZ, P.; SULIK, K. K. Development of neurotransmitter systems in the mouse embryo following acute ethanol exposure: a histological and cytochemical study. International Journal of Developmental Neuroscience. v.8, p.507-522, 1990.

SCHUCKIT, M. A. Alcohol y Alcoholismo. In: KASPER D. L.; FAUCI A.S.; LONGO D. L. Harrison Principios de Medicina Interna. 16º edición. México: Mac Graw Hill, 2005.

SELLERS, E. M.; TONEATTO, T.; ROMACH, M.K.; Somer, G. R.; Sobell, L. C.; Sobell, M. B. Clinical efficacy of the 5$\mathrm{HT}_{3}$ antagonist ondansetron in alcohol abuse and dependence. Alcoholism: Clinical and Experimental Research. v.18, p. 879-885, 1994.

SINGH, L.; WOODRUFF, G. N. CCK Antagonists: pharmacology and applications to drug abuse. Alcohol. v.1, p.27, 1992.

SEITZ, H. K.; STICKEL, F. Molecular mechanisms of alcohol mediated carcinogenesis. Nature Review Cancer, v. 7, p. 599-612, 2007.

SHANK, R. P.; GARDOCKI, J. F.; STREETER, A. J.; MARYANOFF, B. E. An overview of the preclinical aspects of topiramate: pharmacology, pharmacokinetics, and mechanism of action. Epilepsia. v.41(suppl 1), p.S3-9, 2000.

SHETTY, A. K.; BURROWS, R. C.; PHILLIPS, D. E. Alterations in neuronal developmente in the subtantia nigra pars compacta following in utero ethanol exposure inmunohistochemical and Golgi studies. Neuroscience. v.52, n.2, p.311322, 1993.

SPANAGEL, R.; PUTZKE, J.; STEFFERL, A.; SCHOBITZ, B.; ZIEGLGANSBERGER, W. Acamprosate and alcohol. II. Effects on alcohol withdrawal in the rat. European Journal of Pharmacology. v.305, p.45-50, 1996.

SPEAR KING, A. L.; EGIDIO, N. A.; SANTOS, C.M. Risco de suicídio em pacientes alcoolistas com depressão. Jornal Brasileiro de Psiquiatria. v. 55, n.1, p.70-73, 2006. 
STREISSGUTH, A. P.; BARR, H. M.; MARTIN, D. C.; HERMAN, C. S. Effects of maternal alcohol, nicotine, and caffeine use during pregnancy on infant mental and motor development at eight months. Alcoholism: Clinical and Experimental Research. v.4, p.152-164, 1980.

SUVILLAN, E.V. Human brain vulnerability to alcoholism: Evidence from neuroimaging studies. NIAAA Research Monograph No.34. In Review of NIAAA's Neuroscience and Behavioral Research Portfolio, Noronha A.; Eckardt M.; Warren K. p. 473-508, 2000.

TAVERNA, S.; SANCINI, G.; MANTEGAZZA, M.; FRANCESCHETTI, S.; AVANZINI, G. Inhibition of transient and persistent $\mathrm{Na}+$ current fractions by the new anticonvulsant topiramate. Journal of Pharmacology and Experimental Therapeutics. v.288, p.960-968, 1999.

VASCONCELOS, S. M. M.; CALVACANTE, R. A.; AGUILAR, L. M.; SOUSA, F. C. F.; FONTELES, M. M. F.; VIANA, G. S. B. Effects of chronic ethanol treatment on monoamine levels in rat hippocampus and striatum. Brazilian Journal of Medical and Biological Research. v. 37, n.12, p.1839-1846, 2004.

VASCONCELOS, S. M. M. Efeitos Comportamentais, Neuroquímicos e Bioquímicos do etanol em roedores na presença e na ausência de antagonistas dopaminérgicos, glutamatérgicos e opióide. 2001. 245 p. Tese (Doutorado). Programa de Pós-graduação em Farmacologia. Universidade Federal do Ceara. Fortaleza, CE-Brasil.

VOLPICELLI, J. R.; ALTERMAN, A. I.; HAYASHIDA, M.; O'BRIEN, C. P. Naltrexone in the treatment of alcohol dependence. Archives of General Psychiatry. v.49, n.11, p. 876-880, 1992.

WESTERMEYER, J. Historical and social context of psychoactive substance disorders. In: FRANCIS R.; MILLER S. eds. Clinical Textbook of Additive Disorders. New York, Guilford, p. 23-40, 1991.

WEST, J. R.; HAMRE, K. M.; CASSELL, M. D. Effects of ethanol exposure during the third trimester equivalent on neuron number in rat hippocampus and dentate gyrus. . Alcoholism: Clinical and Experimental Research. v.10, p.190$197,1986$.

WILDE, M. I.; WAGSTAFF, A. J.; Acamprosate: A review of its pharmacology and clinical potential in the management of alcohol dependence after detoxification. Drugs. v.53, n. 1038-1053, 1997.

WHITE, H. S.; BROWN, S. D.; WOODHEAD, J. H.; SKEEN, G. A.; WOLF, H. H. Topiramate enhances GABAmediated chloride flux and GABA-evoked chloride currents in murine brain neurons and increases seizure threshold. Epilepsy Research. v.28, p.167-79, 1997.

WHITE, H. S.; BROWN, S. D.; WOODHEAD, J. H.; SKEEN, G. A.; WOLF, H. H. Topiramate modulates GABA-evoked currents in murine cortical neurons by a nonbenzodiazepine mechanism. Epilepsia. v.41(suppl 1), p.S17-20, 2000.

ZALEWSKA-KASZUSKA, J.; GORSKA, D.; DYR, W.; CZARNECKA, E. Effect of acute administration of ethanol on $\beta$ endorphin plasma level in ethanol preferring and non-preferring rats chronically treated with naltrexone. Pharmacology Biochemistry \& Behavior. v.85, p.155-9, 2006.

ZHANG, X.; VELUMIAN, A. A.; JONES, O.T.; CARLEN, P. L. Modulation of high-voltage-activated calcium channels in dentate granule cells by topiramate. Epilepsia. v.41(suppl 1), p.S52-60, 2000.

ZHOU, F. C.; SARI, Y.; POWROZEK, T.; GOODLETT, C. R.; LI, T. K. . Moderate Alcohol exposure compromises neural tube midline development in prenatal brain. Developmental Brain Research. v.144, n.1, p. 43-55, 2003.

National Institute on Alcohol Abuse and Alcoholism. http://www.niaaa.nih.gov. Data do acesso: 29-07-2007. 